\title{
Comment situer les emplois dénominatif et métonymique des noms propres par rapport à leur emploi référentiel et aux noms communs? - les cas de la coordination nominales
}

\author{
Makoto Kaneko* \\ Faculté des Lettres, Université Aoyama Gakuin, 4-4-25 Shibuya, Shibuya-ku, Tokyo 150-8366, Japon
}

\begin{abstract}
Résumé. Cet article se propose d'examiner l'emploi dénominatif des noms propres (e.g. Alfred dans «Il y a trois Alfred dans cette classe. ») et leur emploi métonymique (e.g. Rodin dans "Paul a acheté un Rodin. ») afin de vérifier les hypothèses suivantes avancées par certains travaux récents: A) les deux emplois sont dérivés de l'emploi référentiel (e.g. Paul dans l'exemple précédent) et, tout en disposant d'une certaine «prédicativité » commune aux noms communs, ils doivent être distingués de ces derniers ; B) la dérivation des deux emplois est fondée sur différents principes : l'emploi métonymique est construit à travers «l'interprétation différée » de l'emploi référentiel, tandis que l'emploi dénominatif est établi au moyen du «changement catégoriel». Il est montré que ces hypothèses sont étayées i) par six tests proposés dans une série d'études de Georges Kleiber dans laquelle il préconise une différence sémantique fondamentale entre les noms propres et les noms communs, ainsi que ii) par cinq tests ici proposés en termes de coordination intersective ou non intersective mettant en jeu un ou deux nom(s) propre(s). Les tests en termes de coordination permettent par ailleurs de situer l'emploi métonymique dans une position plus proche des noms communs que l'emploi dénominatif.
\end{abstract}

\begin{abstract}
How to locate denominative and metonymical uses of proper names with respect to their referential use and to commons nouns?- cases of nominal coordination. This article aims at examining denominative use of proper names (e.g. Alfred in "There are three Alfreds in this class. ») and their metonymical use (e.g. Rodin in «Paul bought a Rodin.») in order to verify the following hypotheses advanced by some recent studies: A) both of the two uses are derived from the ordinary referential use (e.g. Paul in the previous example) and, although bearing a certain 'predicativity' similar to common nouns, they should be distinguished from the latter; B) the derivation of the two uses is based on different principles. Thus, the metonymical use is construed through 'deferred interpretation' of the referential use, while the denominative one is established by means of 'category change'. It is shown that these hypotheses are supported i) by six tests provided by a series of studies of Georges Kleiber who advocates a fondamental semantic difference between proper names and common nouns, as well as ii) by five tests proposed here in terms of intersective or non-intersective coordination including one or two proper name(s). The tests in terms of the coordination further allow us to locate metonymical proper names in a position nearer to common nouns than denominative ones.
\end{abstract}

\section{Introduction}

La présente étude se propose d'apporter un nouvel éclairage sur la relation entre les noms communs et les noms propres, en examinant notamment les emplois «dénominatif» et «métonymique » de ces derniers : dans l'emploi dénominatif, les noms propres ne dénotent pas le seul individu, à la différence de l'emploi référentiel ordinaire (illustré par Paul en (1b)), mais dénotent un ensemble d'individus qui ont pour propriété commune de porter le même nom, comme Alfred Muller en (1a); dans l'emploi métonymique, ils dénotent une «entité unie au porteur du nom par une certaine relation » (Kleiber $1992: 186$ ). Ainsi Rodin en (1b) représente une statue fabriquée par l'artiste ${ }^{1}$.

(1)a. Il y a trois Alfred Muller dans le village². (Kleiber $1981: 332$ ) [dénominatif]

b. Paul a acheté un Rodin. (Kleiber $1992: 188$ ) [métonymique]

La discussion est principalement fondée sur les données du français (tirées de travaux antérieurs et recueillies sur Internet, notamment dans Frantext, ou fabriquées par moi-même) mais aussi sur celles du japonais où les deux emplois sont morphologiquement distingués par différents classificateurs (cf. Downing 1996). Par exemple, en (2a), qui contient l'emploi dénominatif du nom propre Alfred, le classificateur pour personne nin est utilisé avec le numéral, tandis qu'en (2b) et en (2c), qui mettent en jeu les emplois métonymiques de Monet et Balzac, sont utilisés deux autres classificateurs, ten et satu, qui sont respectivement spécifiés pour les œuvres d'art et pour les livres.

\footnotetext{
${ }^{*}$ Auteur de correspondance : kanekomakoto06@gmail.com
} 


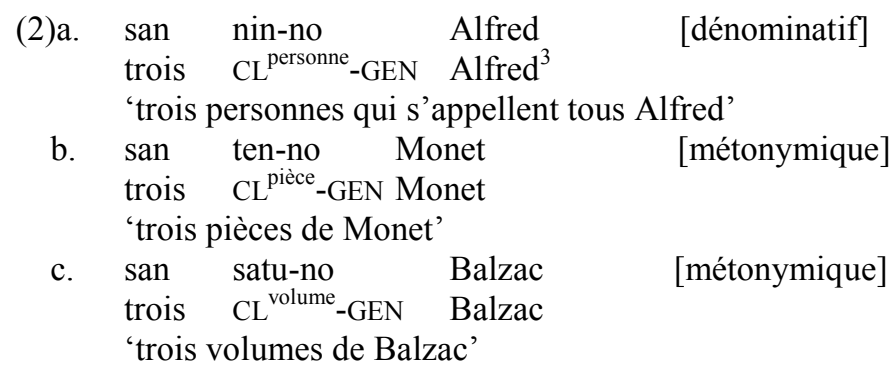

Les principales hypothèses défendues dans cet article (qui sont avancées dans certains travaux récents sur la sémantique des noms propres) sont résumées en $(3 a, b)$. Dans ce qui suit, ces hypothèses seront appuyées par i) certaines données introduites dans une série d'études de Georges Kleiber, et par ii) certains tests ici proposés en termes de coordination mettant en jeu un ou deux nom(s) propre(s). Il sera aussi montré que l'examen des cas de coordination nous permet de comparer la mise en position des deux emplois des noms propres par rapport aux noms communs.

(3)a. Les emplois dénominatif et métonymique sont dérivés de l'emploi référentiel et, tout en disposant d'une certaine 'prédicativité' commune aux noms communs, ils doivent être distingués de ceux-ci.

b. Leurs dérivations sont basées sur différents principes : l'emploi métonymique est construit à travers 'l'interprétation différée' ('deferred interpretation' chez Nunberg 2004) de l'emploi référentiel, tandis que l'emploi dénominatif est établi au moyen du 'changement catégoriel'.

La discussion de cet article est structurée comme suit. Dans la section 2, je passerai d'abord en revue le débat récent sur la sémantique des noms propres, qui me permet de dégager les hypothèses en $(3 \mathrm{a}, \mathrm{b})$. Je présenterai ensuite une série d'études de Georges Kleiber dont l'idée essentielle s'avère compatible avec ces hypothèses. Puis, après avoir discuté, dans la section 3, certaines propriétés de la coordination nominale en français, j'envisagerai, dans la section 4, certains cas de coordination incluant un ou deux nom(s) propre(s), ce qui non seulement viendra appuyer les hypothèses de (3a.b), mais indiquera à quel point la dérivation de chacun des deux emplois aboutit. La section 5 récapitulera les résultats de cet article tout en évoquant les difficultés qui demeurent.

\section{Travaux antérieurs}

Dans cette section, je passerai d'abord en revue le débat récent sur la sémantique des noms propres (2.1). J'examinerai ensuite une série de travaux de Georges Kleiber qui visent à mettre en relief la différence fondamentale entre les noms propres dénominatif et métonymique et les noms communs, tout en acceptant leurs points communs (2.2). La soussection 2.3 récapitulera les résultats de la section 2 .

\subsection{Référentialisme (Asémantisme) vs. Prédicativisme}

Pour la sémantique des noms propres, deux perspectives opposées ont été avancées : Référentialisme (Asémantisme) et Prédicativisme. En gros, le premier soutient que les noms propres n'ont pas de sens (non extentionnel), qu'ils sont sémantiquement des constantes individuelles (cf. Mill 1843) et qu'ils sont des désignateurs rigides qui désignent le même objet dans tous les mondes possibles (Kripke 1980). Il est bien connu que cette approche rencontre des difficultés à traiter des exemples comme en (1a) incluant l'emploi dénominatif ainsi que (4a-d) qui impliquent quatre lectures : quantifiée, non-spécifique, de variable liée et d'incorporation.

(4)a. Il y aura de moins en moins d'Émile en France. (Kleiber 1981 : 334) [lecture quantifiée]

b. Je veux épouser une Albertine. - En as-tu trouvé une ? (ibid.) [non-spécifique]

c. If a child is christened 'Bambi', then Disney will sue Bambi's parents. (Geurts $1997: 321$ ) [variable liée]

d. This is a Bronx-type environment. (Borer $2005: 84$ )

e. $\quad[[$ Alfred $]]=\lambda \times\left(x\right.$ est appelé /Alfred/) (adapté de Kleiber $\left.1981: 326^{4}\right)$

Pour rendre compte de ces exemples, le Référentialisme serait obligé de supposer, contre l'intuition, qu'il s'agit d'une homonymie : par exemple, dans le cas de trois Alfred Muller en (1a), trois personnes ont différents noms propres spécifiques, mais partagent en commun le nom propre générique ${ }^{5}$ (cf. Kaplan 1990). En revanche, ces exemples sont naturellement expliqués par le Prédicativisme qui analyse les noms propres en termes de prédicat, «être appelé /X/», comme le signale (4e). Comme le note Fara (2015 : 251), le Prédicativisme «tente de traiter les noms propres dans une position prédicative comme ayant la même valeur sémantique qu'un nom commun ${ }^{6}{ }^{6}$

Le traitement parallèle entre l'emploi dénominatif et le nom commun est étayé par les données suivantes du niuéen (langue océanique appartenant aux langues polynésienne et qui est parlée en Niue, un pays insulaire de l'océan Pacifique sud). D’après Ghomeshi \& Massam (2019 : 9), cette langue dispose de différents articles pour les noms propres et les noms communs. Par exemple, un nom propre, comme Maro, prend, dans une position casuelle absolutive, un article $a$ comme en (5a), tandis qu'un nom commun, comme tama ('enfant'), prend, dans la même position, un autre article $e$, comme en (5b). Or, lorsqu'un nom propre, comme Mele, est pluralisé et utilisé de façon dénominative, comme en $(5 \mathrm{c})$, il ne prend pas l'article spécifié pour le nom propre $a$, mais celui du nom commun $e$. 
(5)a. déterminant absolutif + nom propre référentiel

a Malo ('Malo') (Ghomeshi \& Massam 2019 : 9)

b. déterminant absolutif + nom commun

e tama ('enfant') ( ibid.)

c. Loga tau Mele he makete ni nai. (idem.10-11)

être.nombreux DET $^{\text {absolutif }} P L$ Marie dans marché plus tôt

'Il y avait beaucoup de Marie au marché plus tôt le matin.'

Le Prédicativisme tente par ailleurs de rendre compte de tous les emplois du nom propre en termes de prédicat «être appelé $/ \mathrm{X} / »$ : ainsi, dans l'emploi référentiel aussi, les noms propres sont des prédicats accompagnés soit d'un démonstratif implicite (Burge 1973), soit d'un article défini implicite (Kleiber 1981, Geurts 1997, Borer 2005, Matushansky 2007, Ghomeshi \& Massam 2009, Izumi 2012, entre autres).

Cette explication unificatrice est pourtant mise en cause par le fait qu'en niuéen, un nom propre référentiel et un nom commun sont accompagnés de différents articles, comme en (5a) et (5b). Un contraste analogue est aussi observé en catalan, où un nom commun masculin singulier, comme noi ('garçon'), prend l'article défini $e l$, comme en (6a), tandis qu'avec un nom propre masculin singulier, comme Joan, un autre article défini, en, est utilisé, comme en (6b).

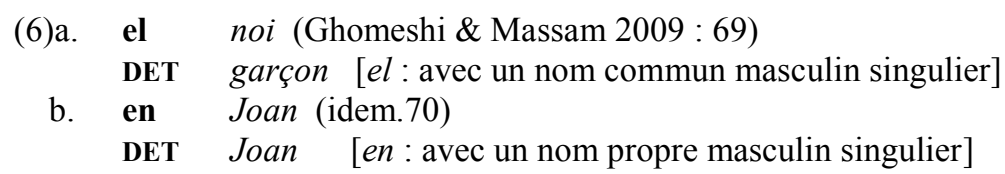

Comme le fait remarquer Jeshion (2015a), l'emploi métonymique est aussi un problème pour l'analyse unificatrice : le nom propre Rodin en (7) ne semble pas être analysé en termes de prédicat «être appelé /Rodin/», mais d'un autre prédicat « être liée à Rodin » ou plus précisément « être une statue fabriquée par Rodin ».

(7) Paul a acheté un Rodin. (=(1b))

(8)a. The ham sandwich is sitting at table seven. (Nunberg $1993: 42$ )

b. Put the gorillas in the east wing and the humans in the west wing. (Fara $2015: 260$ )

Pour défendre le Prédicativisme contre cette critique, Fala (2015) rapproche l'emploi métonymique des noms propres d'une lecture particulière des noms communs que Nunberg (2004) appelle "interprétation différée 》 ('deferred interpretation'). Cette interprétation suppose qu' « une expression réfère à quelque chose qui n'est pas incluse dans la dénotation conventionnelle de cette expression » (Nunberg $2004: 344)^{7}$. Ainsi, le syntagme nominal, the ham sandwich en $(8 \mathrm{a})$, ne réfère pas à un sandwich au jambon, mais est pragmatiquement associé à la personne qui en commande un. De même, d'après Fara (2015 : 260), quand (8b) est énoncé par un curateur de musée d'art à son assistant pour lui indiquer où mettre telle et telle peinture, les deux syntagmes nominaux, the gorillas et the humans, peuvent référer aux œuvres d'art fabriquées respectivement par les gorilles par les êtres humains. Fara (2015) soutient qu'il en est de même pour l'emploi métonymique d'un nom propre, comme en (7) : celui-ci est PRAGMATIQUEMENT dérivé du sens littéral (i.e. l'emploi référentiel) et la sémantique des noms propres ne doit donc pas s'en occuper.

Delgado $(2019: 397 \text {, note } 29)^{8}$ fait toutefois remarquer, suite à Jeshion $(2015 \mathrm{~b})$, une différence importante entre la lecture métonymique de un Rodin en (7) et la lecture différée de the gorillas en (8b) : celle-ci parle des œuvres d'art fabriquées par tous ceux qui satisfont un prédicat « être un gorille » (autrement dit, par n'importe quel gorille), tandis que celle-là ne désigne pas les œuvres d'art fabriquées par tous ceux qui satisfont un prédicat "être appelé /Rodin/ », mais par une unique personne, Auguste Rodin. Ceci suggère que la base de l'emploi métonymique est la lecture référentielle, et non pas la lecture dénominative, comme le suggère le Prédicativisme ${ }^{9}$.

Pour étayer cette analyse, Delgado (2019: 397) rapproche la différence entre l'emploi métonymique d'un nom propre, comme Rodin en (7), et celui d'un nom commun, comme gorillas en (8b), de la différence entre l'emploi verbal d'un nom propre, comme Chriholm en (9a), et celui d'un nom commun, comme bottle en (9b) : « tandis que, dans la conversion d'un nom commun au verbe, comme en $[(9 b)]$, le sens du verbe converti implique le sens non restreint du

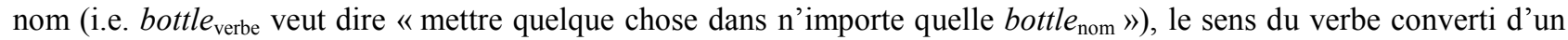
nom propre doit être associé à un individu particulier et non pas à l'ensemble des individus ainsi nommés (e.g. Chriholm). Ceci indique que l'emploi verbal d'un nom propre dépend sémantiquement de son sens référentiel et non pas de son sens prédicationnel ${ }^{10}$. Il en est de même, selon cet auteur, pour l'emploi métonymique d'un nom propre et celui d'un nom commun.

(9)a. I need to Chriholm this definition. (2019:393)

b. Bottle the juice. (idem.391)

D'après Delgado (2019), de la même façon que l'emploi métonymique, l'emploi dénominatif est dérivé de l'emploi référentiel, mais le principe de la dérivation est différent dans les deux cas : la dérivation de l'emploi dénominatif n'est pas basée sur l'interprétation différée, mais sur le «changement catégoriel ». Un autre cas du changement catégoriel est illustré par l'emploi prédicationnel du conjonctif but en anglais, observé en (10).

I have two buts to your proposal. (Delgado $2019: 385$ )

Delgado (2019) suggère aussi que le changement catégoriel est caractérisé par sa nature partielle et son unidirectionnalité. Par exemple, l'emploi prédicationnel du conjonctif but n'est compatible qu'avec un nombre beaucoup 
plus limité de quantificateurs ou déterminants que dans celui d'un nom commun (qui est dès le début d'ordre prédicationnel). Il en est de même pour l'emploi dénominatif d'un nom propre : en anglais, un nom propre singulier n'est pas compatible avec l'article défini sauf s'il est modifié ; en français, un nom propre lui-même ne prend pas la forme plurielle dans son emploi dénominatif même avec un numéral (e.g. trois Alfred vs.*trois Alfreds).

Dans la section suivante, je présenterai une série d'études de Georges Kleiber sur les noms propres fournissant des données empiriques qui, on le verra, s'avèrent compatibles avec i) l'hypothèse de la "partialité » de la dérivation des emplois dénominatif et métonymique (i.e. le fait qu'ils ne sont pas parvenus au statut plein et entier d'un nom commun) et ii) celle de la différence du type dérivationnel des deux emplois (i.e. l'emploi métonymique est construit à travers l'interprétation différée ; l'emploi dénominatif est établi au moyen du changement catégoriel).

\subsection{Kleiber (1981, 1992, 1995, 1996, 2016)}

Dans cette sous-section, j'envisagerai d'abord une différence fondamentale que Kleiber met en relief entre les noms propres et les noms communs (2.2.1). Je présenterai ensuite certaines données que cet auteur mobilise pour illustrer la spécificité des noms propres métonymiques (2.2.2) et celle des noms propres dénominatifs (2.2.3) par rapport aux noms communs.

\subsubsection{Dénomination vs. Catégorisation}

D'après Kleiber, la sémantique des noms propres est fondée sur la dénomination qui consiste à référer à une occurrence particulière, tandis que celle des noms communs, sur la catégorisation consistant à identifier un référent au moyen de son appartenance à une catégorie. Autrement dit, « [les noms communs] constitue(nt) un ensemble sémantique organisé [tandis que] les noms propres [...] ne sont pas intégrés dans le réseau sémantique » (Kleiber 1981 : 404). Cet auteur fait remarquer au moins trois phénomènes qui viennent appuyer la distinction entre la dénomination et la catégorisation.

\section{[1] Disponibilité d'un synonyme ou d'un antonyme}

N'étant pas intégrés dans le réseau sémantique, les noms propres ne nous permettent pas de concevoir de synonyme ou d'antonyme, comme en (11a), à la différence des noms communs, comme en (11b).

(11)a. *Le synonyme de Paul est...

b. Le synonyme de chat est félin.

\section{[2] Référence intercatégorielle}

Tout en admettant que « les noms propres présupposent une catégorisation préalable du particulier qu'ils dénomment », Kleiber (1996 : 581) note qu'ils permettent une référence intercatégorielle à la différence des noms communs. Kleiber (1996 : 570) fait ainsi remarquer qu'en (12), "Marie-Rose peut aussi bien être un nom de personne, de revue, de shampoing contre les poux, de layette ou encore d'un code Minitel type Messagerie rose 3615 ». Il en va de même non seulement pour l'emploi dénominatif, comme en (13a) et (13b), où le nom propre Marie-Rose, accompagné d'un article défini au pluriel, dénote respectivement un ensemble de personnes et un ensemble de shampoings anti-poux, mais aussi pour l'emploi métonymique, comme en (14a), où le nom propre Degas, accompagné d'un numéral, dénote soit une peinture soit une sculpture de danseuse. En japonais, il est possible de recourir à différents classificateurs pour parler de Degas au sens de ses peintures et de Degas au sens de ses sculptures, comme en (14b).

(12) Marie-Rose n'est pas mal. (Kleiber $1996: 570$ )

(13)a. Quand fête-t-on les Marie-Rose (https://www.prenoms.com/prenom/signification-prenom-MARIE-ROSE.html).

b. les Marie-Rose et autres traitements anti-poux (http://annechiffon5.canalblog.com/archives/2008/05/28/9362161.html)

(14)a. Le musée a acheté deux Degas, une des ses peintures et une autre de ses sculptures.
b. ni-mai-no Degas (pour la peinture) / ni-tai-no deux-CL ${ }^{\text {peinture }}$-GEN Degas
$/$ deuX-CL ${ }^{\text {sculpture }}$-GEN
Degas (pour la sculpture)

\section{[3] Catégorisation floue}

L'entité dénotée par un nom commun peut répondre «plus ou moins bien aux critères d'appartenance de catégorie » (Kleiber 2016: 33). Les noms communs sont donc compatibles avec des expressions comme "cette espèce de », comme en (15a), ou « une sorte de », comme en (16a). Ceci n'est pas le cas pour les noms propres pour lesquels « il n'y a pas de degré ou de flou » (ibid.), comme le montre la difficulté de (15b) et (16b).

(15)a. Cette espèce d'écrivain ferait mieux de planter des choux ! (Kleiber $2016: 33$ )

b. ? Cette espèce de Paul ferait mieux de planter des choux ! (ibid.)

(16)a. Un moineau est une sorte d'oiseau. (Kleiber 1981 : 408)

b. ? C'est une sorte de Bernard... (ibid.)

Or, la catégorisation floue est envisageable pour l'emploi métaphorique des noms propres (i.e. antonomase), qui « dénote un type ou une catégorie, dont les membres sont interprétés comme portant une certaine ressemblance avec un particulier bien connu, portant le nom propre en question » (Kleiber 1992 : 188). Ainsi, le nom propre Pierre Bourdieu en (17a) dénote une catégorie consistant en propriétés incarnées par cet individu, et est donc compatible avec un peu qui sert à suggérer l'appartenance incomplète d'un autre individu (en occurrence, Judith Butler) à la catégorie en question. Qui plus est, l'antonomase peut être formée non seulement à partir de l'emploi référentiel des noms propres, comme en (17a), mais aussi de leur emploi métonymique, comme en (17b), où le nom propre Cézanne utilisé métonymiquement 
est compatible avec une sorte de. Ceci indique que l'emploi métaphorique doit être analysé en termes de catégorisation plutôt que de dénomination (cf. Leroy 2004), et doit donc être traité différemment des autres emplois des noms propres.

(17)a. Judith Butler [...] est un peu la Pierre Bourdieu du champ de ces études féministes (Leroy 2004 : 91)

b. Derain et Matisse à Collioure en 1905 exacerbent le sentiment de la présence en portant les couleurs dans l'incandescence, obtenant ainsi une sorte de Cézanne chromatiquement exalté (Jacques Darriulat, Cézanne et la force des choses, http://www.jdarriulat.net/Essais/Cezanne/Cezanne2.html)

En effet, comme le fait remarquer Jeshion $(2015 \mathrm{a}: 230)^{11}$, les emplois dénominatif et métonymique sont normalement classés parmi les emplois «littéraux», tandis que le Référentialisme et le Prédicativisme s'accordent à analyser l'emploi métaphorique comme «non littéral », « spécial» ou «non standard ». Je suis ici cette distinction admise dans les deux écoles pour mettre à part l'emploi métaphorique.

\subsubsection{Nom propre métonymique vs. nom commun}

Dans la même veine que Fala (2015) qui analyse l'emploi métonymique comme un cas dérivé (plus précisément, pragmatiquement différé) de l'emploi référentiel (voir la section 2.1), Kleiber (1992: 198) affirme que «les noms propres quantifiés, même en emploi métonymique, donc en emploi où le référent n'est plus un/le porteur du nom [...], ne s'émancipent pas jusqu'à accéder au status de véritable nom commun ». Pour justifier cette analyse, il observe au moins deux différences entre les noms propres métonymiques et les noms communs.

\section{[1] Genre grammatical}

Tandis que chaque nom commun a son propre genre grammatical, le nom propre est, dans son emploi métonymique, toujours masculin ${ }^{12}$, indépendamment du type d'objet et du sexe de l'auteur. Ainsi même si l'objet contextuellement identifié est représenté par un nom féminin (e.g. statue), le nom propre Rodin, utilisé métonymiquement, ne peut pas prendre un article indéfini féminin, comme en (18a); de même, on ne peut pas utiliser un article partitif féminin avec Françoise Sagan pour dénoter des pages écrites par cette auteure, comme en (18b).

(18)a. Cette statue, c'est \{*une Rodin / un Rodin\}. (Kleiber 1992 : 198)

b. Je lis $\{*$ de la Françoise Sagan / du Françoise Sagan\}. (ibid.)

Cette restriction sur le genre grammatical milite contre l'analyse en termes d'ellipse ${ }^{13}$, selon laquelle, par exemple, un Monet dans «Le musée a acheté un Monet » serait une forme elliptique de un tableau de Monet.

Il est intéressant de constater qu'à la différence de l'emploi métonymique, le genre grammatical de l'emploi dénominatif est fonction du genre de l'emploi référentiel, comme en (19). Ceci vient étayer les hypothèses de Fara (2015) et de Delgado (2019) selon lesquelles, bien que les deux emplois soient dérivés de l'emploi référentiel, leurs dérivations sont fondées sur différents principes.

(19) Je veux épouser une Albertine. - En as-tu trouvé une ? (=(4b))

(20) Judith Butler [...] est un peu la Pierre Bourdieu du champ de ces études féministes (=(17a))

Qui plus est, l'exemple en (20) indique que le genre grammatical de l'antonomase ne dépend plus de celui de l'emploi référentiel : il ne s'agit donc plus de l'individu originellement dénoté par Pierre Bourdieu, mais d'une catégorie consistant en propriétés qu'évoque ce nom propre (e.g. être renommé hors de son propre domaine, être l'auteur de best sellers, etc.). Le genre grammatical fournit ainsi un argument de plus pour traiter différemment l'antonomase.

\section{[2] Lecture de sous-espèce}

La deuxième différence qu'observe Kleiber entre les noms propres métonymiques et les noms communs concerne la possibilité de la lecture de sous-espèce. Étant situés dans une hiérarchie catégorielle, les noms communs admettent une lecture de sous-espèce : (21a) peut être interprété comme « il y a trois types de chevaux ». Ceci n'est pas le cas pour l'emploi métonymique: (21b) ne signifie pas qu' «il y a trois types de statues de Rodin », ce qui suggère, d'après Kleiber, que l'emploi métonymique dénote, comme l'emploi référentiel, une occurrence individuelle.

(21)a. Il y a trois chevaux. (Kleiber 1992 : 198) [ $\sqrt{ }$ lecture de sous-espèce / لlecture d'occurrence individuelle] $=$ il y a $\{$ trois types / trois occurrences $\}$ de chevaux.

b. Il y a trois Rodin. (ibid.) [*lecture de sous-espèce / Vlecture d'occurrence individuelle] = il y a $\{$ *trois types / trois occurrences $\}$ de statues de Rodin.

\subsubsection{Nom propre dénominatif vs. nom commun}

Kleiber (2016 : 33) soutient, pour l'emploi dénominatif, que «ce nom propre «communisé [...] conserve une trace propriale, puisque les entités qu'il rassemble doivent être elles-mêmes, contrairement aux entités rassemblées par les noms communs, porteuses du nom de la catégorie ». Pour confirmer cette distinction, il propose au moins deux critères.

\section{[1] Apprentissage inféré}

Le premier critère concerne l'apprentissage: «l'apprentissage du nom propre pour son porteur ne permet guère d'utiliser ensuite sans nouvel apprentissage ce nom propre pour une autre entité [tandis que] le nom commun [...] permet, une fois la compétence acquise, d'être utilisé sans nouvel apprentissage pour toute entité qui présente les propriétés attendues [...] de la classe ou catégorie délimitée par le concept en question. » (Kleiber 2016:33) 
Curieusement, l'emploi métonymique se comporte, de ce point de vue, de façon analogue au nom commun : une fois qu'on a acquis les propriétés des œuvres produites par un auteur, on peut identifier une de leurs occurrences sans avoir préalablement connaissance du lien entre le nom propre et l'œuvre en question, comme le montre l'acceptabilité de (22). Ceci suggère que l'emploi métonymique est plus proche des noms communs que l'emploi dénominatif sur ce point.

[Un spécialiste des peintures de Vermeer dit]

Compte tenu des propriétés de cette peinture, je suppose que c'est un Vermeer.

[2] Coordination intersective

Kleiber (1981) fait ensuite remarquer que, tandis que deux noms communs sont intersectivement coordonnés l'un avec l'autre, comme en (23a), un nom propre n'est pas intersectivement coordonné avec un autre nom commun, comme en $(23 b, c)$.

(23)a. Le gardien de but et capitaine de l'équipe a été expulsé. (Kleiber 1981 : 402)

b. *Le Paul et capitaine de l'équipe a été expulsé. (ibid.)

c. *Le gardien de but et Paul a été expulsé. (ibid.)

On ne peut toutefois pas exclure la possibilité que l'unicité du référent empêche la lecture dénominative en (23b,c). J'examinerai, dans la sous-section 4.1, si la coordination intersective continue à être impossible lorsqu'on met au clair la pluralité des référents, non seulement pour l'emploi dénominatif mais aussi pour l'emploi métonymique.

\subsection{Récapitulation}

Dans la section 2, j'ai d'abord présenté l'opposition entre le Référentialisme et le Prédicativisme sur la sémantique des noms propres, au terme duquel apparaissent les hypothèses suivantes.

(24)a. Les emplois dénominatif et métonymique sont dérivés tous deux de l'emploi référentiel et, tout en disposant d'une certaine " prédicativité » commune aux noms communs, ils doivent être distingués de ceux-ci. $(=(3 \mathrm{a}))$

b. Leurs dérivations sont fondées sur différents principes : l'emploi métonymique est construit à travers l'interprétation différée de l'emploi référentiel (cf. Fara 2015), tandis que l'emploi dénominatif est établi au moyen du changement catégoriel (cf. Delgado 2019). (=(3b))

J'ai ensuite montré que les résultats des six tests que propose une série d'études de Kleiber (récapitulés dans le Tableau 1) sont compatibles avec ces hypothèses : le test en termes de genre grammatical confirme notamment l'hypothèse de (24b). Mais n'étant appliqués originellement qu'à l'un des deux emplois, ces critères ne sont pas toujours utiles pour mettre au clair les points communs et les différences de chacun des deux emplois par rapport aux noms communs, comme le montrent les trois cellules barrées dans le Tableau 1.

Tableau 1. Résultats des six tests proposés par Kleiber (1981, 1992, 1995, 1996, 2016)

\begin{tabular}{|l|c|c|c|c|c|c|}
\hline & $\begin{array}{c}\text { référence } \\
\text { intercatégorielle }\end{array}$ & $\begin{array}{c}\text { catégorisa- } \\
\text { tion floue }\end{array}$ & $\begin{array}{c}\text { déterminant } \\
\text { féminin }\end{array}$ & $\begin{array}{c}\text { lecture sous- } \\
\text { espèce }\end{array}$ & $\begin{array}{c}\text { acquisition } \\
\text { inférée }\end{array}$ & $\begin{array}{c}\text { coordination intersective } \\
\text { avec un NC }\end{array}$ \\
\hline $\mathrm{NC}$ & $*$ & $\sqrt{ }(15 \mathrm{a}),(16 \mathrm{a})$ & $\sqrt{ }$ & $\sqrt{ }(21 \mathrm{a})$ & $\sqrt{ }$ & $\sqrt{ }(23 \mathrm{a})$ \\
\hline $\mathrm{NPr}$ métonymique & $\sqrt{ }(13 \mathrm{a}, \mathrm{b})$ & $\sqrt{ }(17 \mathrm{~b})$ & $*(18 \mathrm{a}, \mathrm{b})$ & $*(21 \mathrm{~b})$ & $\sqrt{ }(22)$ & \\
\hline $\mathrm{NPr}^{\text {dénominatif }}$ & $\sqrt{ }(14 \mathrm{a})$ & & $\sqrt{ }(19)$ & & $*$ & $*(23 \mathrm{~b}, \mathrm{c})$, mais au pluriel ? \\
\hline
\end{tabular}

Dans ce qui suit, après avoir examiné, dans la section 3, certaines contraintes imposées sur la coordination nominale, j'envisagerai, dans la section 4 , les cas mettant en jeu une coordination pour comparer plus systématiquement les deux emplois des noms propres avec les noms communs.

\section{Coordination des noms communs en français et en japonais}

En français, un seul déterminant ou un seul numéral peut précéder la coordination intersective des noms communs, comme le prouvent (25a) incluant un déterminant possessif $m a$ ainsi que (25b) impliquant un numéral deux.

(25)a. Un jour, ma voisine et amie me dit [...] (Agota Kristof, L'Analphabète)

b. J'ai rencontré deux collègues et amis (Goosse \& Grevisse 2015, Le Bon Usage, 812).

Pour la coordination non intersective des noms communs, Milner (1979) fait remarquer qu'un seul numéral est difficilement suivi des conjoints nominaux, comme en (26a), tandis que l'acceptabilité augmente quand la coordination est accompagnée, comme en (26b), d'une relative restrictive (soulignée en (26b)).

(26)a. *On a condamné pour menées subversives cinq professeurs de lycée et étudiants de première année. (Milner $1979: 91)$

b. On a condamné pour menées subversives cinq professeurs de lycée et étudiants de première année qui ont signé la pétition. (ibid.) 
Dans la même veine, Roodenburg (2005 : 98) observe qu' « il est difficile en français de coordonner des $\mathrm{N}$ [=nom] en mettant en facteur le déterminant, sauf [...] si ces $\mathrm{N}$ forment un groupe naturel ", comme l'indique (27a), et que la présence d'une relative restrictive (soulignée en (27b)) améliore l'acceptabilité de la coordination avec un seul déterminant, comme en (27b).

(27)a. ??Les locataires et propriétaires se retrouvent dans la cour tous les dimanches.

b. Les locataires et propriétaires qui le souhaitent se retrouvent dans la cour tous les dimanches. (Roodenburg $2005: 98)$

Pour rendre compte de l'incompatibilité entre un seul déterminant ou un seul numéral et la coordination des noms communs, je me réfère aux remarques de Jespersen (1924) ${ }^{14}$, de Wierzbicka (1988) ${ }^{15}$ et de Dostie (2015). Jespersen et Wierzbicka s'accordent à soutenir que compter les membres d'un ensemble exige l'uniformité de ceux-ci, et qu'il n'est donc possible de compter les entités que si celles-ci sont de la même espèce. De même, Dostie $(2015$ : 163) suggère qu'un seul déterminant ne précède la coordination que lorsque celle-ci désigne des " entités prises de manière unifiée ", comme frères et sœurs dans « Avez-vous des frères et sœurs?».

Suivant ces analyses, je soutiendrai que la détermination effectuée au sein du syntagme nominal ainsi que la quantification numérique exigent l'uniformité des membres dénotés, et que les deux séquences «cinq professeurs de lycée et étudiants de première année » en (26a) et «les locataires et propriétaires » en (27a) sont difficilement acceptées à cause de la non uniformité des référents des conjoints.

Cette analyse est indirectement appuyée sur le fait qu'en japonais, qui dispose des classificateurs, la séquence correspondante à « cinq professeurs de lycée et étudiants de première année » en (26a) est acceptable, comme en (28). C'est parce que le classificateur pour personne nin sert à recatégoriser des membres a priori différents, en mettant en avant leur dénominateur commun et en mettant en suspens la différence lexicale.

$\begin{array}{llll}\text { go-nin-no } & \begin{array}{l}\text { [kookoo-no } \\ \text { lycée-GEN }\end{array} \text { professeur et un-an-GEN étudiant } \\ \text { cinq-CL } & \text { personne-GEN } & \begin{array}{l}\text { lycé } \\ \text { 'cinq personnes consistant en professeurs de lycée et étudiants de premier année' }\end{array}\end{array}$

On peut maintenant rendre compte de l'effet de la relative restrictive, observé en (26b) et (27b), comme suit. D'après Gary-Prieur (2001), la relative restrictive établit une catégorisation discursive. Cette catégorie contextuellement construite met en suspens la différence de catégorisation lexicale (ex. professeurs de lycée vs. étudiants de première année) et sert, comme un classificateur, à fournir une unité aux membres a priori hétérogènes. Cette analyse s'accorde avec une remarque de Milner (1979) selon laquelle « les deux N' conjoints désignent un groupe 'mixte' dont l'unité est indiquée dans la relative ».

\section{Coordination mettant en jeu un ou deux nom(s) propre(s)}

Après avoir ainsi examiné les propriétés de la coordination nominale, j’aborderai maintenant les cas de la coordination incluant un ou deux nom(s) propre(s). Au terme de la discussion, on verra que les tests en termes de coordination non seulement confirmeront les hypothèses de $(24 a, b)$ (voir la section 2.4$)$, mais aussi nous permettront de comparer la mise en position des noms propres dénominatif et métonymique par rapport aux noms communs.

\subsection{Coordination intersective}

Je commencerai par examiner la coordination intersective entre un nom propre et un nom commun, déjà discutée par Kleiber (1981) (voir la section 2.2.3). La coordination en (29a) implique un nom propre métonymique et un nom commun, tandis que celle en $(29 \mathrm{~b})$ met en jeu un nom propre dénominatif et un nom commun. Certains de mes informateurs trouvent (29a) plus acceptable que (29b). Ceci indique qu'au moins pour eux, un nom propre métonymique est plus proche d'un nom commun qu'un nom propre dénominatif dans cet environnement.

(29)a. Ce musée possède deux estampes de Hokusai qui composent les 36 vues du Mont Fuji. ? Ces deux Hokusai et trésors du musée attirent plus de 100 mille visiteurs chaque année. [DET $+\mathrm{NUM}+\mathrm{Npr}$ métonymique $^{\mathrm{NC}}$ ]

b. J'ai deux amis qui habitent dans le même arrondissement et qui portent d'ailleurs le même nom, François. *Je rends visite de temps en temps aux deux François et voisin ${ }^{16}$. [DET $\left.+\mathrm{NUM}+\mathrm{Npr}^{\text {dénominatif }}+\mathrm{NC}\right]$

\subsection{Coordination non intersective}

Puis, en ce qui concerne la coordination non intersective, j'examinerai d'abord les cas consistant en un nom propre et un nom commun (4.2.1) et ensuite les cas contenant deux noms propres du même emploi (4.2.2).

\subsubsection{Nom propre métonymique + nom commun vs. nom propre dénominatif + nom commun}

En (30a), un seul déterminant et un seul numéral sont suivis de la coordination consistant en un nom propre métonymique et un nom commun; en $(30 \mathrm{~b})$, ils sont suivis de celle d'un nom propre métonymique et d'un nom commun. Selon mes informateurs, (30a) n'est pas acceptable si le numéral quatre porte sur l'ensemble des deux pièces de Hokusai et des deux trésors nationaux du Japon. De même (30b) n'est pas acceptable si le numéral cinq compte l'ensemble des quatre membres de Kravehl et du violoncelliste. Ces résultats ne sont pas surprenants d'autant que même deux noms communs ne sont pas factorisés par un seul numéral, comme on l'a vu dans la section 3 (voir 
l'exemple (26a)).

(30)a. Cette exhibition présente deux estampes de Hokusai et deux peintures qui sont des trésors nationaux du Japon.

*Ces quatre Hokusai et trésors du Japon attirent plus de 10 mille visiteurs chaque semaine.

$\left[\mathrm{DET}+\mathrm{NUM}+\mathrm{Npr}^{\text {métonymique }}+\mathrm{NC}\right]$

b. Les quatre membres du Quatuor Kravehl sont des frères et soeurs et le nom du quatuor vient de leur nom de famille. Ils jouent parfois avec leur violoncelliste préféré. L'autre jour, *Ies cinq Kravehl et violoncelliste ont joué le quintette à cordes de Schubert. [DET $\left.+\mathrm{NUM}+\mathrm{Npr}^{\text {dénominatif }}+\mathrm{NC}\right]$

Même en japonais, où le classificateur permet à un seul numéral de factoriser deux noms communs lexicalement différents (voir l'exemple (28)), un seul numéral n'est compatible ni avec la coordination d'un nom propre métonymique et d'un nom commun, ni avec celle d'un nom propre dénominatif et d'un nom commun, dans l'interprétation pertinente, comme en $(31 \mathrm{a}, \mathrm{b})$. Selon mes informateurs japonais, le numéral ne porte que sur le premier conjoint dans les deux cas, ce qui confirme la distinction fondamentale entre les noms propres dénominatif et métonymique et les noms communs.

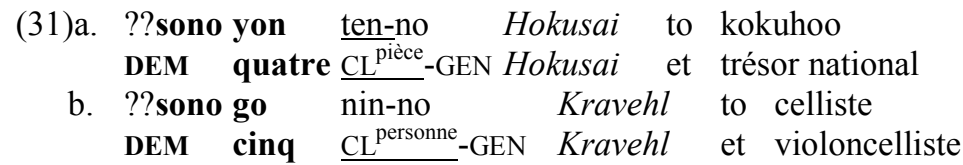

Or, l'acceptabilité de (32a) suggère qu'il n'est pas impossible qu'à la différence d'un numéral, un seul déterminant factorise un nom propre métonymique et un nom commun. Ceci n'est pas le cas pour la coordination consistant en un nom propre dénominatif et un nom commun, comme le montre l'inacceptabilité de (32b). Cette différence suggère qu'un nom propre métonymique se comporte d'une façon plus analogue à un nom commun qu'un nom propre dénominatif dans cet environnement.

(32)a. Cette exhibition présente deux estampes de Hokusai et deux peintures qui sont des trésors nationaux du Japon

Ces Hokusai et trésors nationaux attirent plus de 10 mille visiteurs chaque semaine. [DET $+\mathrm{Npr}^{\text {métonymique }}+\mathrm{Nc}$ ]

b. Les quatre membres du Quatuor Kravehl sont des frères et soeurs et le nom du quatuor vient de leur nom de famille. Ils jouent parfois avec leur violoncelliste préféré. L'autre jour, *les Kravehl et violoncelliste ont joué le quintette à cordes de Schubert. [DET $\left.+\mathrm{Npr}^{\text {dénominatif }}+\mathrm{NC}\right]$

Il est aussi à noter que la différence d'acceptabilité entre (30a) et (32a) indique que i) l'exigence de l'uniformité ne joue pas de la même manière pour les déterminants définis et les quantificateurs, et ii) la cardinalité est plus sensible à l'homogénéité des membres ${ }^{17}$.

\subsubsection{Deux noms propres avec ou sans relative restrictive}

Examinons pour finir les cas de coordination de deux noms propres du même type. Les exemples en (33a) et (33b) impliquent respectivement deux noms propres métonymiques et deux noms propres dénominatifs. Dans les deux cas, la coordination est introduite par un déterminant défini et un numéral et suivie d'une relative restrictive. D'après mes informateurs, les deux exemples sont acceptables, ce qui constitue un point commun avec la coordination de deux noms communs (voir (26b) et (27b) dans la section 3).

(33)a. Les quatorze Monet et Cézanne qui se trouvent dans ce musée sont tous magnifiques. $\left[\mathrm{DET}+\mathrm{NUM}+\right.$ deux $\mathrm{Npr}^{\text {métonymique }}+$ relative restrictive $]$

b. En français, Camille et Dominique peuvent dénoter un garçon ou une fille. Mais les cinq Camille et Dominique que je connais sont toutes des filles. [DET $+\mathrm{NUM}+\operatorname{deux} \mathrm{Npr}^{\text {dénominatif }}+$ relative restrictive]

Or, sans relative restrictive, la factorisation de deux noms propres métonymiques au moyen d'un seul déterminant est en principe exclue, mais parfois possible, comme en (34a). Ceci ne semble pas être le cas avec deux noms propres dénominatifs sauf si ceux-ci forment un «duo $»^{18}$, comme le montre la difficulté de (34b) (il est à noter que Degas et Whistler en (34a) ne semblent pas former un duo). Une fois encore, ce test indique que l'emploi métonymique est plus proche du nom commun que l'emploi dénominatif.

(34)a. [...] incline devant les Monet, génufléchis devant les Degas et Whistler (É. Benveniste, Convergences typologiques, 1966 : Frantext) [DET + deux $\mathrm{Npr}^{\text {métonymique }}$ ]

b. J'ai rencontré des Guermantes et *(des) Courvoisier chez la princesse de Parme. [DET + deux Npr $\left.{ }^{\text {dénominatif }}\right]$

\subsection{Récapitulation}

Les résultats de la section 4 sont récapitulés dans le Tableau 2. Ces résultats indiquent que i) les noms propres métonymique et dénominatif montrent certes des différences par rapport aux noms communs, mais aussi des points communs pour les tests $\mathrm{A}$ et $\mathrm{E}$, et ii) les noms propres métonymiques se situent dans une position intermédiaire entre les noms communs et les noms propres dénominatifs pour le test $\mathrm{D}$. 
Tableau 2. Résultats de cinq en termes de coordination mettant en jeu un ou deux nom(s) propre(s).

\begin{tabular}{|c|c|c|c|c|c|}
\hline & $\begin{array}{c}\text { A. } N U M[X 1 \text { et X2]+restrictive } \\
\text { (non intersectif) }\end{array}$ & $\begin{array}{c}\text { B. } \text { DET }[X 1 \text { et X2] } \\
\text { (non intersectif) }\end{array}$ & $\begin{array}{c}\text { C.DET }[X \text { et } N C] \\
\text { (non intersectif) }\end{array}$ & $\begin{array}{c}\text { D. } N U M[X \text { et } N C] \\
\text { (intersectif) }\end{array}$ & $\begin{array}{c}\text { E. } N U M[X \text { et } N C] \\
\text { (non intersectif) }\end{array}$ \\
\hline $\mathrm{NC}$ & $\sqrt{ }(26 \mathrm{~b}),(27 \mathrm{~b})$ & $? ?(27 \mathrm{a})$ & $? ?(27 \mathrm{a})$ & $\sqrt{ }(25 \mathrm{~b})$ & $*(26 \mathrm{a})$ \\
\hline $\mathrm{Npr}^{\text {métonymique }}$ & $\sqrt{ }(33 \mathrm{a})$ & $*$, mais $\sqrt{ }(34 \mathrm{a})$ & $\sqrt{ }(32 \mathrm{a})$ & $?(29 \mathrm{a})$ & $*(30 \mathrm{a})$ \\
\hline $\mathrm{Npr}^{\text {dénominatif }}$ & $\sqrt{ }(33 \mathrm{~b})$ & $*(34 \mathrm{~b})$ & $*(32 \mathrm{~b})$ & $*(29 \mathrm{~b})$ & $*(30 \mathrm{~b})$ \\
\hline
\end{tabular}

\section{Remarques récapitulatives et problèmes qui restent}

Dans cet article, j'ai commencé par présenter, au terme de l'examen sur le récent débat portant sur la sémantique des noms propres, les hypothèses suivantes.

(35)a. Les emplois dénominatif et métonymique sont tous deux dérivés de l'emploi référentiel et, tout en disposant d'une certaine « prédicativité » commune aux noms communs, ils doivent être distingués de ceux-ci. $(=(24 \mathrm{a}))$

b. Leurs dérivations sont fondées sur différents principes: l'emploi métonymique est construit à travers l'interprétation différée de l'emploi référentiel (cf. Fara 2015), tandis que l'emploi dénominatif est établi au moyen du changement catégoriel (cf. Delgado 2019). (=(24b))

J'ai ensuite montré que ces hypothèses sont appuyées par les six tests que propose une série d'études de Kleiber (examinés dans la section 2.2 et récapitulés dans le Tableau 1) et par les cinq tests ici proposés en termes de coordination (discutés dans la section 4 et récapitulés dans le Tableau 2). Les tests de la coordination nous ont par ailleurs permis de situer les noms propres métonymiques dans une position intermédiaire entre les noms communs et les noms propres dénominatifs.

Certaines questions demeurent. D'abord, suivant les analyses précédentes, cet article a traité à part l'emploi métaphorique des noms propres : celui-ci doit être analysé, pour parler comme Kleiber, en termes de la catégorisation, parallèlement aux noms communs, ce qui est confirmé notamment par sa compatibilité avec la catégorisation floue (voir la section 2.2.1). La coordination non intersective fournit un autre argument pour rapprocher l'antonomase du nom commun. Selon Leroy (2004 : 91-92), «l'article défini pluriel peut parfois regrouper deux référents étroitement associés [e.g. (36a,b)]. [...] Cette construction du nom propre en antonomase [...] semble avant tout liée au « duo » des deux référents originels des noms propres $»$.

(36)a. Le domestique amoureux. Zhao Bang et Ziba, les Tristan et Iseut du Yi-king, tu vois ? (Daniel Pennac, Aux fruits de la passion, 1999 : Frantext)

b. Nous sommes les Roméo et Juliette de ce milieu. (Leroy 2004 : 92)

Il semble possible de supposer que les cas de l'antonomase du duo (ou du couple) tels qu'en (36a,b) correspondent aux cas de la coordination des deux noms communs, précédée d'un seul déterminant, qui représentent « un hyperonyme construit extensionnellement par conjonction de deux ou plusieurs hyponymes » (Benetti 2008 : 93), comme pins et sapins en (37a), ou une expression idiomatique, comme frères et sœurs en (37b) (Michaux 1998: 325). Il reste à examiner si les autres cas de la coordination viennent également à l'appui du rapprochement entre l'antonomase et le nom commun.

(37)a. Les pins et sapins avaient conservé leur ramure qui se renouvelle de saison en saison sans se dépouiller jamais (Benetti 2008 : 91)

b. Il connaît très bien les frères et sæurs du Premier Ministre. (Michaux 1998 : 325)

Cet article n'a pas abordé l'emploi de 'fractionnement' des noms propres. Il s'agit de l'emploi dans lequel le référent « ne représente plus qu'une partie, un aspect, une manifestation » du porteur d'un nom propre (Kleiber 1992 : 187). Or, la coordination des noms communs et celle des noms propres de fractionnement manifestent une distribution analogue. Roodenbourg (2005), entre autres, fait remarquer que la coordination des noms communs est en principe incompatible avec un seul déterminant, qu'elle peut apparaître, avec certains types de prédicat verbal, sans déterminant, comme en (38a), et que la présence d'un modifieur restrictif exige celle d'un seul déterminant, comme en (38b) (voir la discussion dans la section 3).

(38)a. Locataires et propriétaires se retrouvent dans la cour tous les dimanches.

b. Les locataires et propriétaires qui le souhaitent se retrouvent dans la cour tous les dimanches. $(=(27 \mathrm{~b}))$

(39)a. France et Allemagne ont connu des évolutions contraires en ce qui concerne le taux de pauvreté relative [...] http://www.ladocumentationfrancaise.fr/var/storage/rapports-publics/114000118.pdf

b. Les France et Allemagne des années 2000 ont connu des évolutions contraires en ce qui concerne le taux de pauvreté relative.

Or, deux noms propres qui ne forment pas nécessairement un « duo », comme France et Allemagne en (39a), peuvent être coordonnés sans déterminant. Avec un modifieur servant à délimiter le référent spatio-temporellement, comme des années 2000, souligné en (39b), les noms propres sont employés de manière fractionnée et exigent la présence d'un seul déterminant. Il serait alors intéressant d'examiner comment l'usage du fractionnement se comporte par rapport aux autres tests mettant en jeu la coordination. 


\section{Références bibliographiques}

Acquaviva, P. 2019. Nouns as names, names as nouns. Exemplier d'une communication donnée au colloque «Le nom, entre syntaxe et sémantique », Université de Paris-Sorbonne, 29 mars 2019.

Benetti, L. (2008). L'article zéro en français contemporain : aspect syntaxique et sémantique. Bern : Peter Lang.

Borer, H. 2005. Structuring Sense vol.1 In Name Only. Oxford: Oxford University Press.

Brisson, C. (2003). Plural, all, and the nonuniformity of collective predication. Linguistics \& Philosophy, 26, 129-184.

Burge, T. (1973). Reference and proper names. The Journal of Philosophy, 70(14), 425-439.

Delgado, L. 2019. Between singularity and generality : the semantic life of proper names. Linguistic \& Philosophy, 42, 381-417.

Dostie, G. (2015). Réflexions sur la (quasi-)synonymie et la variation diaphasique. In G. Dostie \& P. Hadermann (éds.). La diavariation en français actuel. Bern : Peter Lang, 147-177.

Downing, P. (1996). Numeral classifier systems: the case of Japanese. Amsterdam: John Benjamins.

Fara, D. (2015). “Literal” Uses of Proper Names. In A. Bianchi (éd.). On Reference. Oxford: Oxford University Press, 251-279.

Gary-Prieur, M.-N. (1994). Grammaire du nom propre. Paris : PUF.

Gary-Prieur, M.-N. (2001). GN démonstratif à référence générique : une généralité discursive. French Language Studies, 11, 221239

Geurts, B. (1997). Good news about the description theory of names. Journal of Semantics, 14, 319-348.

Ghomeshi, J. \& D. Massam (2009). The proper D connection. In J. Ghomeshi, I. Paul \& M. Wiltschko (éds.). Determiners: Universals and variation. Amsterdam: John Benjamins, 67-98.

Ghomeshi, J. \& D. Massam (2019). Number Is Different in Nominal and Pronominal Phrases. Linguistic Inquiry, 1-14. https://doi.org/10.1162/ling a 00350

Izumi, Y. (2012). The semantics of proper names and other bare nominals. Doctoral Dissertation, University of Maryland.

Jespersen, O. (1924). The philosophy of grammar. London: G. Allen \& Unwin, ltd.

Jonasson, K. (1994). Le Nom propre. Constructions et interprétations. Louvain-la-Neuve : Duculot.

Jeshion, R. (2005a). Names Not Predicates. In A. Bianchi (éd.). On Reference. Oxford: Oxford University Press, 225-250.

Jeshion, R. (2005b). A Rejoinder to Fara's "Literal" Uses of Proper Names. In A. Bianchi (éd.). On Reference. Oxford: Oxford University Press, 280-294.

Kaplan, D. (1990). Words. Proceedings of the Aristotelian Society, 64, 93-119.

Kleiber, G. (1981). Problèmes de référence: descriptions définies et noms propres. Paris : Klincksieck.

Kleiber, G. (1992). Quand le noms propre prend article : le cas des noms propres métonymiques. Journal of French Language Studies, 2, 185-205.

Kleiber, G. (1995). Sur la définition des noms propres : une dizaine d'années après. In M. Noailly (éd.). Noms propres et nomination. Paris : Klincksieck, 11-36.

Kleiber, G. (1996). Noms propres et noms communs : un problème de dénomination, Meta, 41(4), 567-589.

Kleiber, G. (2016). Noms propres : dénomination et catégorisation. Langue française, 190, 29-44.

Kripke, S. (1980). Naming and Necessity. Boston: Harvard University Press.

Leroy, S. (2004). De l'identification à la catégorisation : l'antonomase du nom propre en français. Louvain-Paris : Éditions Peeters.

Matushansky, O. (2008). On the linguistic complexity of proper names. Linguistics \& Philosophy, 21, 573-627.

Michaux, C. (1998). Reducing the Coordination of Determiners: Some Principle. In F. Hamm \& E. Hinrichs (éds.). Plurality and Quantification. Dordrecht: Kluwer, 321-337.

Mill. J.-S. (1843). A system of logic, ratiocinative and inductive : being a connected view of the principles of evidence, and method of scientific investigation. London : J.W. Parker.

Milner, J.-C. (1979). De la syntaxe à l'interprétation. Paris : Seuil.

Nunberg, G. (1993). Indexicality and Deixis. Linguistics \& Philosophy, 16, 1-43.

Nunberg, G. (2004). The Pragmatics of Deferred Interpretation. In L. Horn \& G. Ward (éds.). Handbook of Pragmatics. Oxford: Blackwell, 344-364.

Roodenburg, J. (2005). Une coordination particulière : les syntagmes $N$ Conj $N$ en français. Languages, 160, 93-109.

Wierzbicka, A. (1988). The Semantics of Grammar. Amsterdam : John Benjamins.

Une partie de cette étude a été présentée au colloque "Le nom, entre syntaxe et sémantique », organisé par Université de ParisSorbonne, 28-30 mars 2019. Je remercie les participants de leurs remarques précieuses. Je suis également reconnaissant à Anne 
Zribi-Hertz et mes collègues d'Aoyama pour nos échanges, leur jugement d'acceptabilité et la correction du français. Toutes les insuffisances qui restent sont dues à l'auteur. Cette étude a reçu le soutien financier de la Japan Society for the Promotion of Sciences (numéro 18k00536).

${ }^{1}$ Cet article ne traitera pas de l'emploi métaphorique des noms propres (appelé aussi « antonomase »), illustré en (i). Cette exclusion sera justifiée à la fin de la section 2.2.1.

(i) Judith Butler [...] est un peu la Pierre Bourdieu du champ de ces études féministes (Leroy 2004 : 91)

${ }^{2}$ Dans les exemples cités, le nom propre sera mis en italique, et le déterminant et /ou le numéral qui l'introduit, en gras.

${ }^{3}$ Les abréviations utilisées dans cet article sont les suivantes $: \mathrm{CL}=$ classificateur $; \mathrm{DEM}=$ démonstratif $; \mathrm{DET}=$ déterminant ; GEN $=$ génitif $; \mathrm{NC}=$ nom commun ; $\mathrm{Npr}=$ nom propre $; \mathrm{NUM}=$ numéral $; \mathrm{PL}=$ pluriel

${ }^{4}$ Kleiber (1995) abandonne toutefois l'idée de 'prédicat' dénominatif, et redéfinit la sémantique des noms propres en termes de sens instructionnel.

${ }^{5}$ Acquaviva (2019) tente de sophistiquer une telle analyse. Tout en rendant compte de la sémantique des noms propres en termes de prédicat, être appelé, et en supposant un déterminant implicite, cet auteur soutient que leur partie nominale dénote une espèce abstraite (abstract kind), identifiée mais non pas décrite, et que l'individu nommé est un argument relié à cette espèce abstraite. Ainsi, la sémantique d'un nom propre comme Alex est représentée par (i), où la personne en question (signalée par [[he]]) est reliée par la relation d'appellation (signalée par naming) à une espèce abstraite d'Alex (signalée par [[Alex $]]^{k}$, le signe $k$ indiquant 'kind')

(i) $\lambda \mathrm{w}\left[(\mathrm{w})\right.$, naming $\left([[\right.$ he $\left.\left.]],[[\text { Alex }]]^{\mathrm{k}}\right)\right]$

D'après Acquaviva, ce qui est rigide (i.e. constant dans tous les mondes possibles) est une relation d'étiquetage ('labelling') entre la forme linguistique (e.g. le signifiant Alex) et l'espèce abstraite qu'elle identifie ; la relation entre l'individu désigné et l'appellation est dépendante du choix d'un monde, comme l'indique l'introduction d'une variable de monde $w$ en (i).

${ }^{6}$ « We want to treat [...] proper names in predicate position as having the same type of semantic value as that of other count nouns, namely the semantic value of predicate » (Fara $2015: 251$ )

${ }^{7}$ « By deferred interpretation [...] I mean the phenomenon whereby expressions can be used to refer to [or be true of] something that isn't explicitly included in the conventional denotation of that expression » (Nunberg $2004: 344$ )

${ }^{8}$ « the new meaning is derived from the unrestricted meaning of the common noun, and not from the meaning of the noun once restricted to a particular denotation » (Delgado $2019: 397$, note 29)

${ }^{9}$ Consciente de ce problème, Fala (2015: 259, note 15) note, concernant l'exemple (i) où two Stella dénote deux peintures de l'artiste Frank Stella, que «this might require that in the Stella examples, the extension of the name 'Stella' have been already contextually narrowed down to one person called Stella. The question is whether we could truly say that there were two Stellas in the museum on the grounds that there was a painting by Frank Stella and also one by Joseph Stella ». Le cas mentionné à la fin de cette citation est illustré par (ii) et (iii). Ceci paraît montrer que, lorsqu'il existe plus d'un artiste qui ont le même nom, les œuvres d'art référées peuvent être associées non seulement à une unique personne, mais aussi aux personnes qui satisfont un prédicat dénominatif « est appelé /X/ », conformément à l'analyse prédicativiste.

(i) Two Stellas are inside the museum. (Jeshion 2015a : 226) [two Stellas = deux peintures de l'artiste Frank Stella]

(ii) Ce musée a acheté trois Peter Bruegel : un est du père et les deux autres sont du fils.

(ii) Trois Bach ont été joués ce soir-là : la première pièce était de Carl Phillipe Emmanuel, et les deux autres, de Jean-Sébastian.

Il faudrait pourtant admettre, comme le fait remarquer Delgado (2019), que les individus dont il s'agit en (i)-(iii) sont préalablement limités beaucoup plus sévèrement que dans les cas de lecture différée d'un nom commun, comme en ( $8 \mathrm{~b})$.

${ }^{10}$ « $[\mathrm{W}]$ hile in noun-to-verb conversions the meaning of the converted verb involves the unrestricted meaning of the noun (i.e.

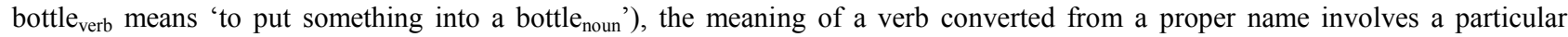
individual but not the set of people called ' $\mathrm{N}$ ', or the property being called ' $\mathrm{N}$ '. This indicates that at least [...] verbal uses semantically depend on the referential meaning of the proper name, and not on the predicative meaning. » (Delgado 2019: 397)

${ }^{11}$ «[The examples like (17a)] are, according to predicativistes, instances of non-literal application of names. Because they are nonliteral uses of proper names, [these examples] are construed by predicativistes as 'special' and 'non-standard' uses that may, and should, receive a different semantic treatment. Almost all theorists, including referentialists, go along with this assumption.»" (Jeshion 2015a : 230)

${ }^{12}$ Certains noms propres originellement métonymiques sont lexicalisés comme des noms communs et sont spécifiés pour différents genres selon le contexte, comme dans les exemples suivants.

(i)a. une Renault $=$ une [voiture] Renault (Kleiber $1992: 189)$

b. un Renault = un [camion ou bus ou tracteur] Renault (ibid.)

${ }^{13}$ Il paraît exister une différence interlinguistique sur le genre grammatical d'un nom propre métonymiquement utilisé. D'après Fala (2015:259, note 16), en allemand, « the indefinite article in 'I bought a Stella' takes the gender of the noun 'painting' (neuter) rather than that of the person Stella (masculine). [...] in 'a Stella [machine]' the article takes the gender of the 'machine', which is feminine. [...] however, the gender behavior of articles with deferrentially interpreted predicates does not generalize to Belgian Dutch. »

${ }^{14}$ «We can only speak of 'more than one' in regard to things which without being identical belong to the same kind [...] if the difference is too great, it is impossible to use words like two or three. » (Jespersen 1924 : 188-189) 
15 «[T]he idea of counting implies not just the presence of separate objects, but the presence of separate objects OF THE SAME KIND. » (Wierzbicka $1988: 512-514$ )

${ }^{16}$ Un des relecteurs fait remarquer que cette phrase devient acceptable sans conjunction de coordination et, comme en «Je rends visite aux deux François voisins. » Le même relecteur trouve par ailleurs l'exemple en (i) plus ou moins acceptable. Mes informateurs préfèrent toutefois l'exemple en (ii) où le nom propre et le nom commun sont apposés.

(i) Les deux François et amis d'enfance / voisins de pallier travaillent au parlement.

(ii) Les deux François, amis d'enfance / voisins de pallier, travaillent au parlement.

${ }^{17}$ Cette différence est également observée dans les cas de la coordination des noms communs, comme le montre le contraste d'acceptabilité suivant.

(i)a. Si vous ne vous présentez pas en début de service, il se peut que vous n'ayez pas de table, les fourchettes et verres sont en bonus. (https://www.tripadvisor.fr/ShowUserReviews-g297949-d317349-r415634056-Sahara Beach Aquapark ResortMonastir Monastir Governorate.html)

b. ??Trois fourchettes et verres sont en bonus.

Effectivement, Brisson (2003) note que les pluriels définis peuvent inclure des membres 'erronés' : ainsi, le pluriel défini, the girls en (ii), peut dénoter un ensemble incluant quelques garçons.

(ii) The girls jumped in the lake. (Brisson $2003: 135$ )

${ }^{18}$ Quand les référents forment un « duo », un seul article défini peut même factoriser deux noms propres référentiels, comme en (i).

(i) [...] eut la gloire de créer les deux Marsis et Girardon (THOMAS Antoine-Léonard, Frantext) 